\title{
Identifying a possible stratification phenomenon in ionospheric F2 layer using the data observed by the DEMETER satellite: method and results
}

\author{
Xiuying Wang, Dehe Yang, Dapeng Liu, and Wei Chu \\ Institute of Crustal Dynamics, China Earthquake Administration, Beijing, China \\ Correspondence: Xiuying Wang (652383915@qq.com) \\ Received: 9 April 2019 - Discussion started: 29 April 2019 \\ Revised: 22 June 2019 - Accepted: 8 July 2019 - Published: 30 July 2019
}

\begin{abstract}
Many studies have revealed the stratification phenomenon of the topside ionospheric F2 layer using groundbased or satellite-based ionograms, which can show direct signs of this phenomenon. However, it is difficult to identify this phenomenon using the satellite-based in situ electron density data. Therefore, a statistical method, using the shuffle resampling skill, is adopted in this paper. For the first time, in situ electron density data, recorded by the same Langmuir probe aboard the DEMETER (Detection of Electro-Magnetic Emission Transmitted from Earthquake Regions) satellite at different altitudes, are analyzed, and a possible stratification phenomenon is identified using the proposed method. Our results show that the nighttime stratification, possibly a permanent phenomenon, can cover most longitudes near the geomagnetic equator, which is not found from the daytime data. The arch-like nighttime stratification decreases slowly on the summer hemisphere and thus extends a larger latitudinal distance from the geomagnetic equator. All results, obtained by the proposed method, indicate that the stratification phenomenon is more complex than what has previously been found. The proposed method is thus an effective one, which can also be used in similar studies of comparing fluctuated data.
\end{abstract}

\section{Introduction}

Stratification of the F2 layer, an enhancement in electron density at heights above the F2-layer maximum in the ionosphere at low latitudes and mid-latitudes, was first reported in the mid-20th century (Heisler, 1962; Sen, 1949; Skinner et al., 1954). Sayers et al. (1963) was then the first to de- tect topside ledges in the equatorial ionosphere using a Langmuir probe aboard the Ariel I satellite and predicted that the topside ionograms would reveal the ledges as cusps, as later proved by many studies using the topside sounding technique (Lockwood and Nelms, 1964; Raghavarao and Sivaraman, 1974; Sharma and Raghavarao, 1989).

There were few studies of the stratification phenomenon until the mid-1990s. Balan and Bailey (1995) then explained the formation mechanism of the F3 layer using the SUPIM (Sheffield University Plasmasphere-Ionosphere Model). They referred to the layer as $\mathrm{G}$ layer, which was later renamed as F3 layer because it has the same chemical composition as the F region (Balan et al., 1997). Since then, many more studies on the mechanism and spatial and temporal distributions of the phenomenon have been carried out (Batista et al., 2002; Depuev and Pulinets, 2001; Hsiao et al., 2001; Rama Rao et al., 2005; Tardelli et al., 2016; Uemoto et al., 2007; Zain et al., 2008; Zhao et al., 2011a, b).

However, most research has used ionograms or total electron content data recorded on the ground (Balan et al., 1998; Batista et al., 2002; Jenkins et al., 1997; Nayak et al., 2014; Rama Rao et al., 2005; Zhao et al., 2011a), where the distribution features of the stratification phenomenon cannot be obtained because only data of discontinuously distributed observation stations can be used. Studies on the stratification of the F2 layer at the topside ionosphere were therefore carried out using sounding techniques aboard low-Earthorbiting satellites (Karpachev et al., 2012; Thampi et al., 2005; Uemoto et al., 2004, 2006; Zhao et al., 2011b). Topside ionograms can reveal the occurrence of the F3 layer when the peak electron density of the F3 layer, namely $N m \mathrm{~F} 3$, is smaller than $N m \mathrm{~F} 2$, which cannot be observed using an 
ionosonde on the ground. However, the short-term globalscale distribution of the stratification phenomenon still cannot be obtained from satellite-based ionograms even though such ionograms can provide more data because the obtained data are still discontinuous.

In addition, nearly all the abovementioned F2-layer stratification studies were carried out using indirect observation data, in which case some detailed information may be missed. A method therefore is proposed in this paper which can compare the in situ electron density data obtained at different altitudes and identify their differences. Based on this method, the in situ electron density data, recorded by the DEMETER (Detection of Electro-Magnetic Emission Transmitted from Earthquake Regions) satellite at the topside ionosphere, are used to study the stratification phenomenon, enabling us to investigate the characteristics of the globalscale distribution and other information about the stratification phenomenon.

The result that the electron density observed at higher altitude is greater than that observed at lower altitude suggests a stratification phenomenon distributed in a large area. This result was obtained using in situ electron density data obtained before and after an altitude adjustment of the DEMETER satellite in a relatively short time, which is the first direct comparison of in situ data recorded by the same instrument but at different altitudes. The results of the distribution features of this phenomenon, obtained by the proposed method, are in accordance with those obtained by previous studies, but some features also suggest that the stratification phenomenon is more complicated than previously found, thus demonstrating that the proposed method is effective.

\section{Data and method}

\subsection{Data}

The data used in this study were obtained from DEMETER, a French micro-satellite operated by CNES (Centre National d'Etudes Spatiales) and devoted to the investigation of ionospheric disturbances due to seismic, volcanic, and tsunami activities. The DEMETER satellite was launched in June 2004. Observation data were recorded from the end of November 2004 to December 2010. Owing to its specific orbit, DEMETER is always located at about 10:30 or $22: 30$ local time. The satellite made continuous measurements between invariant latitudes of -65 and $65^{\circ}$. The ISL (Instrument Sonde de Langmuir) is one of the five scientific payloads and recorded in situ data of the electron density, ion density, and electron temperature (Lagoutte et al., 2006; Lebreton et al., 2006).

The DEMETER satellite adjusted its flying altitude in its initial flight stage and between the end of 2005 and the beginning of 2006, as shown in Fig. 1, which presents the average flight altitude of the ascending (nighttime) and descending (daytime) orbit between southern and northern geographical latitudes of $50^{\circ}$ from 17 November 2004 to 31 December 2006.

The history of the altitude of the satellite can be divided into four stages:

1. The altitude of the satellite was not fixed but varied between about 703 and $725 \mathrm{~km}$ from 17 November 2004 to 10 March 2005.

2. The average orbital altitude was fixed at around $709 \mathrm{~km}$ after 10 March 2005.

3. The average altitude was adjusted to approximately $677 \mathrm{~km}$ from 1 to 9 January 2006.

4. The altitude was fixed at an average value of about $669 \mathrm{~km}$ from 14 January 2006.

The data recorded by the DEMETER satellite before and after its altitude adjustment provide an opportunity to study the vertical gradients of electron density in a small height range of the topside ionosphere using in situ electron density data recorded by the same instrument. Since the altitude of the satellite was not fixed at a constant value from November 2004 to March 2005, and there were no data in December 2005, data recorded before and after the adjustment at the beginning of 2006 are selected in the study; during these periods, the orbit altitude was fixed at 677 and $669 \mathrm{~km}$.

The geomagnetic index Dst and the solar activity index F10.7 in January 2006 are presented in Fig. 2. The figure shows geomagnetically quiet days from 1 to 25 January 2006, and the F10.7 index of solar activity before altitude adjustment was roughly equal to or smaller than that after the adjustment. Therefore, data from 1 to 25 January 2006 will be used in this paper because the differences in geomagnetic and solar influences are negligible during this period.

Many studies have shown that the electron density in the F2 layer is characterized by periodic changes in the diurnal, seasonal, annual, and solar activity cycles and fluctuations due to other factors, such as geomagnetic storms and sunspot eruptions. Issues therefore need to be addressed before carrying out this study.

As mentioned above, the local time that the DEMETER Satellite passed over a location was roughly fixed at about 10:30 in the morning and about 22:30 in the evening, which means that diurnal changes in the data can be ignored when comparing the data before and after the altitude adjustment at the same place because the local time is consistent. Another issue, which is the focus of this study, is that when the electron density data are recorded over a relatively short time under quiet observation conditions, say a few days, variations due to the long-term trend in the data (e.g., seasonal and annual variations) can be ignored, which is to say that the data observed in a few days are usually similar to those observed a few days ago. 


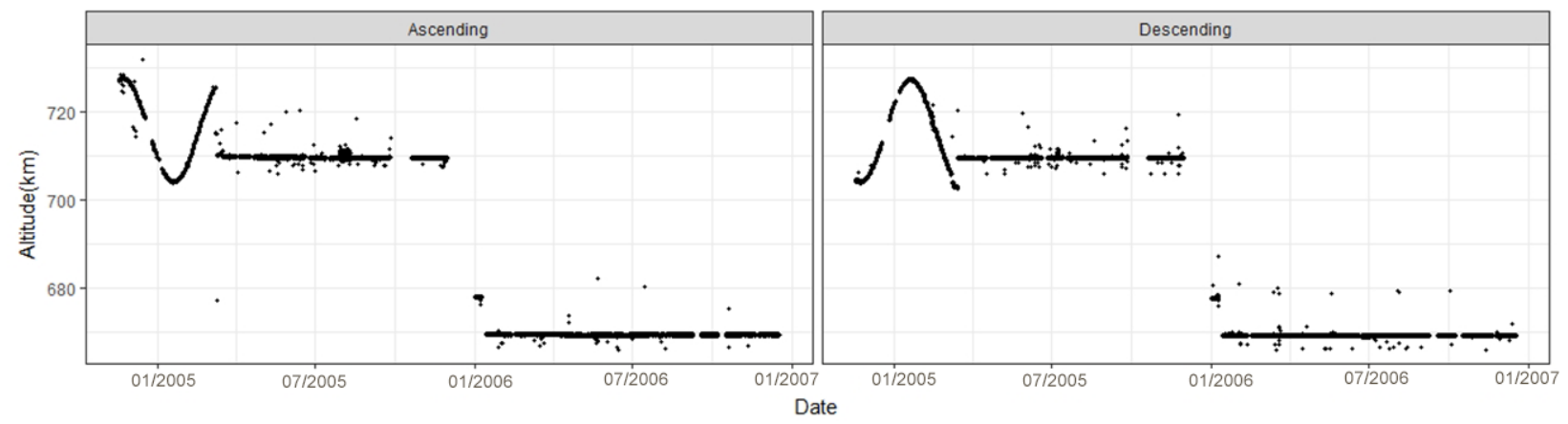

Figure 1. Average altitude of the DEMETER satellite from November 2004 to December 2006.
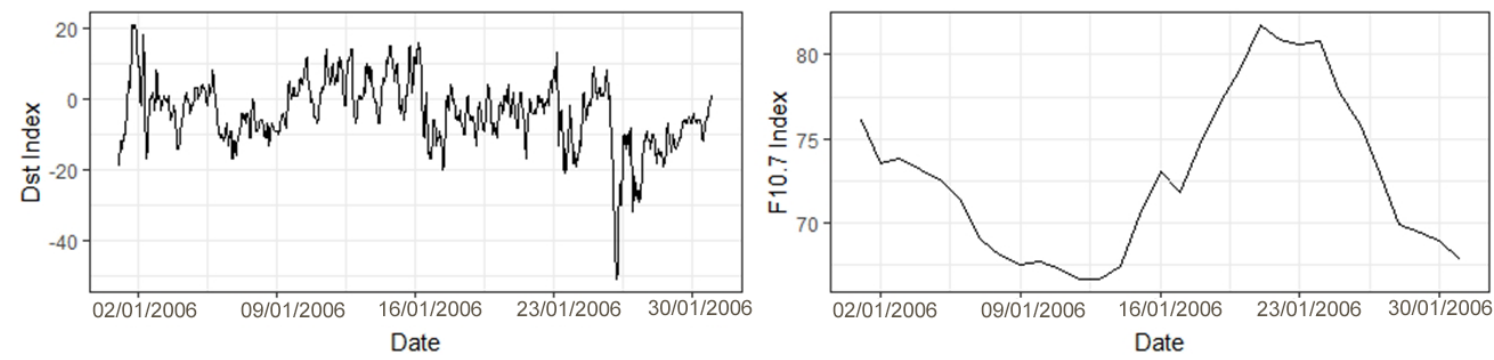

Figure 2. Geomagnetic index Dst and solar activity index F10.7 in January 2006.

Against this background, the data, observed before and after the altitude adjustment of the DEMETER satellite in a relatively very short time, are compared and analyzed by seeking a suitable mathematical method.

\subsection{Method}

The electron density is known to dynamically change both spatially and temporally. It is therefore uncertain that the difference before and after the adjustment of the orbital altitude is the result of normal data fluctuation or the result of the altitude adjustment. It is necessary to design a reasonable scheme with which to distinguish the cause of the difference.

A significance test is a statistical method of determining whether the difference between two groups of data is significant. Employing this method, if the $p$ value, the probability that a given result occurs under the null hypothesis of no difference between the two groups, is less than a predefined significance level, then the null hypothesis is rejected at the chosen level of significance and the alternative hypothesis of a difference between the two groups is accepted. However, if the $p$ value is not less than the chosen significance threshold, then the evidence is insufficient to support a conclusion. Significance tests can therefore be conducted to determine whether the difference between before and after the adjustment of the altitude can be ascribed to the randomness of the data variation. If not, it may be caused by the altitude adjustment because all the other conditions are the same.
However, the significance test assumes data to be normally distributed, which the electron density data are not. This paper thus conducts a permutation test (Hesterberg et al., 2003), a distribution-independent computer simulation approach of resampling suggested by Fisher and Yates (Wikipedia).

The basic idea of the permutation test is to resample the data many times to check whether the same pattern of results is observed if the observation data are randomly assigned to experimental groups. If the statistics calculated from the obtained data fall outside the confidence limits, say $95 \%$, the observed difference is far out in the left or right tail, and one can conclude that there is a significant difference between the groups. A permutation test is based on available data rather than a set of standard assumptions about underlying populations. It is therefore distinct from traditional statistics and can give accurate $p$ values with which to check the significance of the difference between two data groups.

We therefore adopt the permutation test method to compare the data observed at different altitudes by the DEMETER satellite and to check whether the differences between the data observed at different altitudes are significant. Using this method, the general process of data analysis in this study is as follows:

1. Construct data groups using the data observed before and after the altitude adjustment or data observed at same altitude. 
Table 1. Grouping information of the data from 1 to 23 January 2006.

\begin{tabular}{llll}
\hline $\begin{array}{l}\text { Group } \\
\text { go. }\end{array}$ & $\begin{array}{l}\text { Date of } \\
\text { observation }\end{array}$ & $\begin{array}{l}\text { Average } \\
\text { altitude }\end{array}$ & $\begin{array}{l}\text { Altitude } \\
\text { adjustment }\end{array}$ \\
\hline Group 1 & $1,2,3,4$ & $677.76 \mathrm{~km}$ & Before \\
Group 2 & $5,6,7,8$ & $677.78 \mathrm{~km}$ & Before \\
Group 3 & $15,16,17,18$ & $669.34 \mathrm{~km}$ & After \\
Group 4 & $20,21,22,23$ & $669.33 \mathrm{~km}$ & After \\
\hline
\end{tabular}

- Divide the area covered by the satellite orbit between latitudes of $50^{\circ}$ south and $50^{\circ}$ north into cells of $5^{\circ}$ latitude and $10^{\circ}$ longitude.

- Calculate the mean electron density before and after the adjustment of altitude in each cell.

- Divide the data into different regions every $5^{\circ}$ latitude and obtain 20 regions from $50^{\circ}$ south to $50^{\circ}$ north in the latitudinal direction.

2. Compare the data groups constructed from observation at different altitudes and check the significance of their differences by employing the permutation test method.

3. Compare the data groups constructed from observation at similar conditions but with the same altitude and check the significance of their differences as a reference.

4. Draw conclusions by analyzing different results.

A uniform significance level of 0.05 and a one-side test are adopted in this paper, and no special explanation is given in the following.

\section{Data comparison}

\subsection{Data construction}

According to Sect. 2.1, the data obtained from 1 to 25 January 2006 are selected to carry out the analysis. During this period, the data from 1 to 9 January were obtained before the altitude adjustment, and the data from 14 to 25 January were obtained after the altitude adjustment. In addition, the geomagnetic and solar activity indices were all low during this period; that is, the data obtained before and after the altitude adjustment were measured under similar observation conditions.

In order to construct the data groups for comparison, a scheme is designed to divide the data into different groups. Ascending data (data recorded during the night) from 1 to 8 January and from 15 to 23 January 2006 are both divided into two groups to give a total of four groups of data, with each having equal observation days. Details of the grouping are given in Table 1.
Based on this grouping scheme, comparative data are constructed using the cells of $5^{\circ}$ in the latitudinal direction and $10^{\circ}$ in the longitudinal direction, as mentioned in Sect. 2.2. The average value of the recorded data in each cell is computed using data from Group 1 to Group 4; there are thus 36 cells multiplied by 4 groups of data for each latitudinal region. Data analysis involves comparing the data between groups in each latitudinal region, including both the cases of data comparisons between different altitudes and between the same altitudes.

\subsection{Comparison in one latitudinal region}

The four groups of data, in the region of geographical latitude -5 to $0^{\circ}$, are compared with each other as a demonstrative example of the proposed method.

In order to determine whether the differences between two groups of data are caused by random data fluctuation or by altitude differences, significance tests are carried out for each pair of groups using the improved Fisher-Yates permutation test method (Durstenfeld, 1964), in which the distribution of the mean data difference is obtained by resampling the data 10000 times. The actual mean data differences of each pair of groups are then compared with the $5 \%$ confidence level of the corresponding distribution.

The significance test results of each pair of groups using the data located in the geographical latitude $(-5,0)$ are shown in Fig. 3, and the corresponding permutation test $p$ values are given in Table 2 .

In Fig. 3, the solid lines represent mean values of data differences before and after the altitude adjustment in each cell:

$$
\begin{aligned}
M_{\text {Diff }} & =\frac{1}{N} \sum_{i=1}^{N}\left(B_{i}-A_{i}\right) \\
& =\frac{1}{N} \sum_{i=1}^{N} B_{i}-\frac{1}{N} \sum_{i=1}^{N} A_{i} .
\end{aligned}
$$

Here, $N$ is total number of cells in each latitudinal region, $B$ is the average value in cell $i$ before altitude adjustment, and $A$ is the average value in the same cell after the adjustment. Equation (1) shows that the mean value of data differences is equal to the data difference between average values of all cells before and after the adjustment. Therefore, mean values of data differences can be calculated using two average values. As shown in Fig. 3, the data differences, between the average data in the two groups in random permutation tests conducted 10000 times, follow a normal distribution with a mean value of zero, and the probability of the occurrence of the original data difference is zero or extremely small, which indicates that data recorded before the adjustment in most cells are obviously greater than those recorded after the adjustment because the mean differences are much greater than zero.

Figure 3 and Table 2 show that the differences between Group 1 and 3, Group 2 and 3, Group 1 and 4, and Group 2 and 4 , representing the differences before and after the ad- 

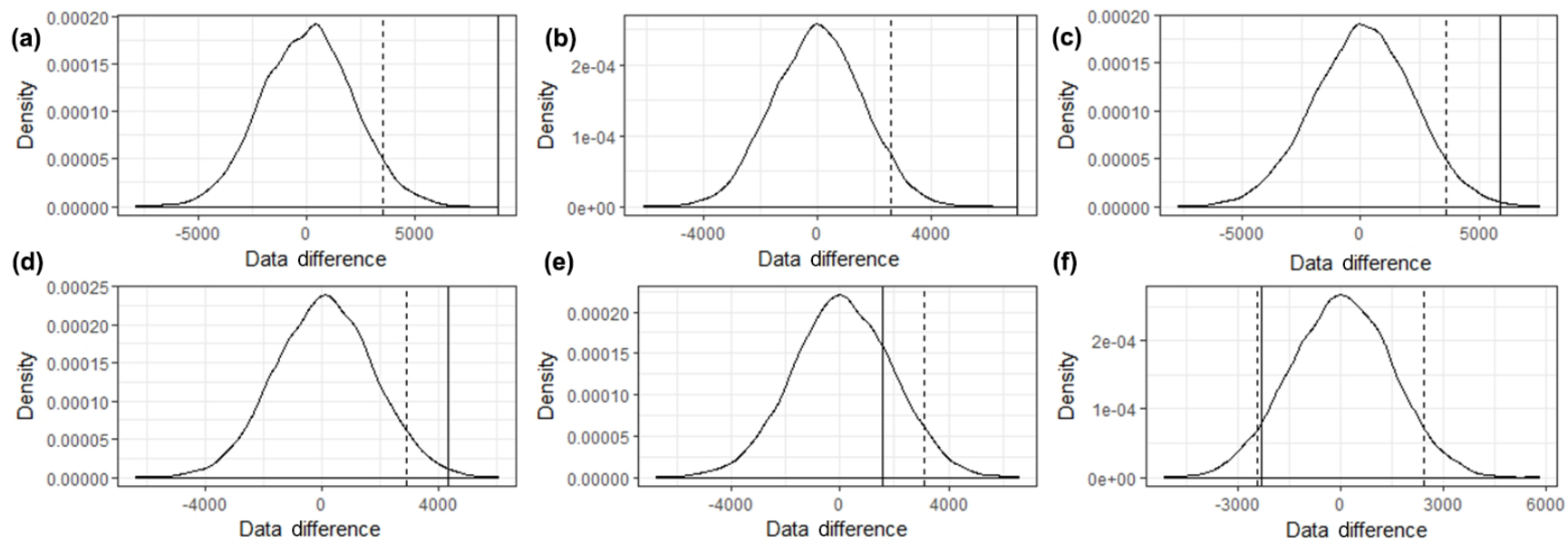

Figure 3. Density distributions of the mean difference obtained in the permutation test. (The dashed line is the mean difference corresponding to the $5 \%$ confidence level, while the solid line is the observed mean difference between two groups. The lower $5 \%$ confidence level is also shown for (f) because the data difference is negative. Here, (a) is the permutation test result for Group 1 and 3, (b) Group 2 and 3, (c) Group 1 and 4, (d) Group 2 and 4, (e) Group 1 and 2, and (f) Group 3 and 4.)

Table 2. Permutation test results of ascending data at a geographical latitude of -5 to $0^{\circ}$.

\begin{tabular}{|c|c|c|c|c|c|c|c|c|c|c|c|c|}
\hline \multirow{2}{*}{$\begin{array}{l}\text { Latitudinal } \\
\text { region }\end{array}$} & \multicolumn{2}{|c|}{ Group 1-3 } & \multicolumn{2}{|c|}{ Group 2-3 } & \multicolumn{2}{|c|}{ Group 1-4 } & \multicolumn{2}{|c|}{ Group 2-4 } & \multicolumn{2}{|c|}{ Group 1-2 } & \multicolumn{2}{|c|}{ Group 3-4 } \\
\hline & $M_{\text {Diff }}$ & $p$ & $M_{\text {Diff }}$ & $p$ & $M_{\text {Diff }}$ & $p$ & $M_{\text {Diff }}$ & $p$ & $M_{\text {Diff }}$ & $p$ & $M_{\text {Diff }}$ & $p$ \\
\hline$(-5,0)$ & 8797.95 & 0.0000 & 7031.11 & 0.0000 & 5909.50 & 0.0025 & 4325.02 & 0.0049 & 1584.48 & 0.2136 & -2312.43 & 0.0593 \\
\hline
\end{tabular}

$M_{\text {Diff }}$ represents the mean of differences between two groups, while $p$ is the probability that the mean data difference calculated in the permutation simulation is greater than the observed $M_{\text {Diff }}$ if it is positive or less than the observed $M_{\text {Diff }}$ if it is negative.

justment of altitude, are significant because the $p$ values are zero or close to zero, much less than the predefined significance level of $5 \%$. This means that the likelihood of observing the actual data difference given that the two groups have no difference is unlikely. Therefore, the null hypothesis of no difference can be rejected, and significant difference between the two groups is determined. Meanwhile, the $p$ values of Group 1 and 2 and Group 3 and 4, representing differences at the same altitude before and after the adjustment respectively, are greater than the predefined significance level, which means that the difference between the two groups is not significant and the hypothesis of no difference between the two groups cannot be rejected.

The permutation test results of data at different altitudes and data at similar altitudes show a significant contrast, indicating that the significant differences between the data before and after the adjustment are by no means accidental but due to potential causes. Moreover, an interesting point is that the electron density data recorded at higher altitude are higher than those at a lower altitude because all differences (i.e., values before adjustment minus values after adjustment) are positive, different from the normal attenuation law at the topside ionosphere, which implies the possible stratification phenomenon during the selected time segment.

\subsection{Comparison in all latitudinal regions}

Obvious differences between the data groups in one latitudinal region show some information. To obtain the distribution of this significant difference, permutation test results for the 20 regions from $50^{\circ}$ south to $50^{\circ}$ north in the geographical and geomagnetic latitude (where the geomagnetic latitude refers to the dipole coordinates given in the DEMETER satellite dataset) are obtained, and the variations in $p$ values with latitude are presented in Fig. 4. Table 3 only gives the permutation test results in geomagnetic latitudes because the results calculated from geographical latitudes are similar to those of geomagnetic latitudes.

The permutation test results in Fig. 4 and Table 3 have obvious regular distribution patterns:

1. There are significant differences in data only before and after the adjustment of altitude in continuous latitudinal regions; i.e., there are significant differences in data between Group 1 and 3, Group 2 and 3, Group 1 and 4, and Group 2 and 4. Meanwhile, the differences between observation data for the same orbital altitude, namely differences between Group 1 and 2 and Group 3 and 4, are not obvious, and no regular distribution pattern exists in the data. 

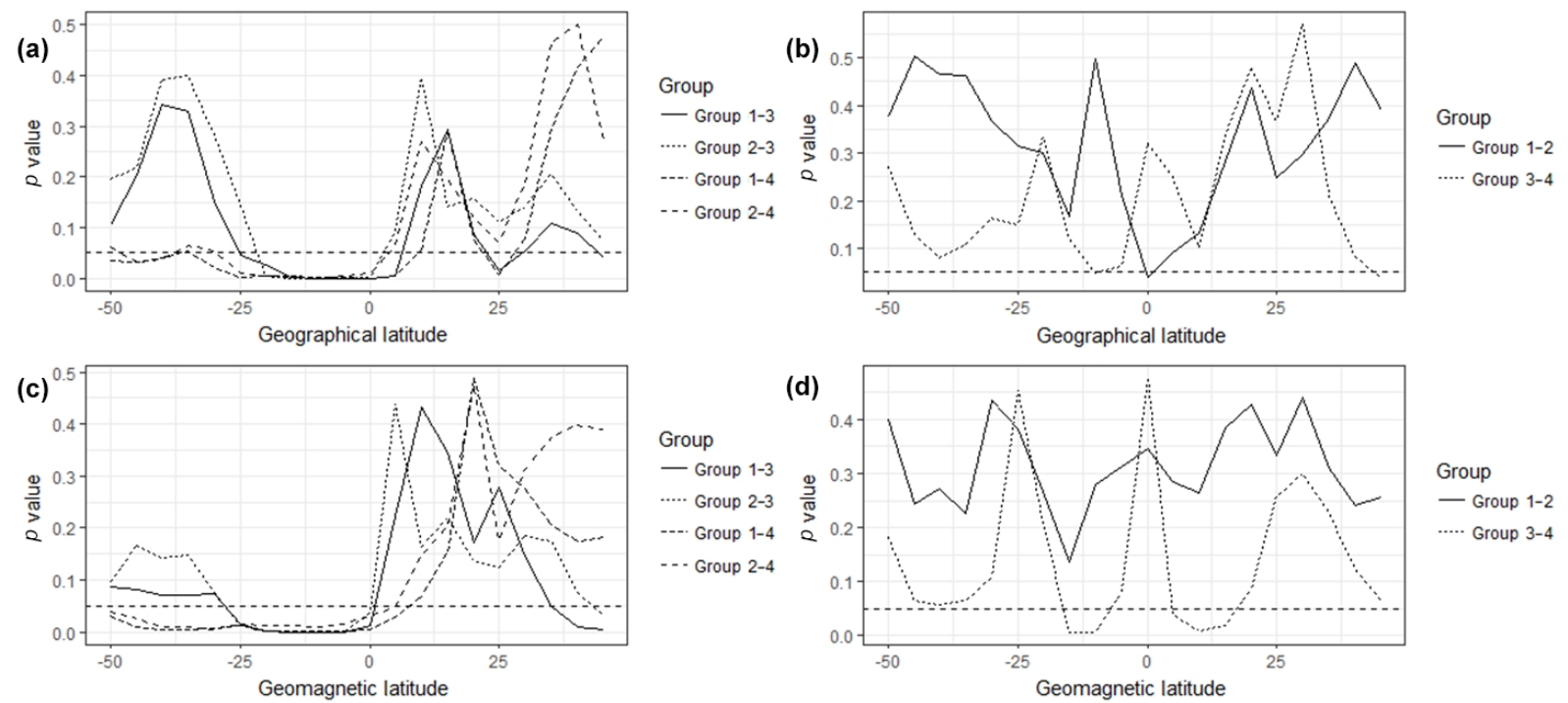

Group

- Group 1-2

.... Group 3-4

Figure 4. Variations in $p$ values with geographical and geomagnetic latitude.

Table 3. Permutation test results of ascending data in the 20 geomagnetic latitudinal regions.

\begin{tabular}{|c|c|c|c|c|c|c|c|c|c|c|c|c|}
\hline \multirow{2}{*}{$\begin{array}{l}\text { Latitudinal } \\
\text { region }\left({ }^{\circ}\right)\end{array}$} & \multicolumn{2}{|c|}{ Group 1-3 } & \multicolumn{2}{|c|}{ Group 2-3 } & \multicolumn{2}{|c|}{ Group 1-4 } & \multicolumn{2}{|c|}{ Group 2-4 } & \multicolumn{2}{|c|}{ Group 1-2 } & \multicolumn{2}{|c|}{ Group 3-4 } \\
\hline & $M_{\text {Diff }}$ & $p$ & $M_{\text {Diff }}$ & $p$ & $M_{\text {Diff }}$ & $p$ & $M_{\text {Diff }}$ & $p$ & $M_{\text {Diff }}$ & $p$ & $M_{\text {Diff }}$ & $p$ \\
\hline 45,50 & -2258.44 & 0.0040 & -1717.80 & 0.0299 & -839.74 & 0.1835 & -299.10 & 0.3889 & -540.64 & 0.2569 & 1418.71 & 0.0639 \\
\hline 40,45 & -1950.41 & 0.0091 & -1335.83 & 0.0770 & -903.82 & 0.1739 & -289.24 & 0.3966 & -614.58 & 0.2418 & 1046.59 & 0.1243 \\
\hline 35,40 & -1184.69 & 0.0507 & -810.07 & 0.1748 & -718.42 & 0.2075 & -343.80 & 0.3748 & -374.62 & 0.3093 & 466.27 & 0.2284 \\
\hline 30,35 & -868.94 & 0.1464 & -770.15 & 0.1845 & -473.54 & 0.2745 & -378.75 & 0.3120 & -98.79 & 0.4401 & 279.48 & 0.2990 \\
\hline 25,30 & -578.48 & 0.2779 & -822.49 & 0.1246 & -372.14 & 0.3199 & -585.42 & 0.1744 & 213.29 & 0.3327 & 324.59 & 0.2562 \\
\hline 20,25 & -901.29 & 0.1727 & -975.20 & 0.1385 & -28.67 & 0.4882 & -72.08 & 0.4747 & 43.41 & 0.4284 & 966.15 & 0.0866 \\
\hline 15,20 & -600.00 & 0.3450 & -986.37 & 0.2207 & 1347.41 & 0.1533 & 961.04 & 0.2034 & 386.37 & 0.3848 & 1947.41 & 0.0185 \\
\hline 10,15 & -269.15 & 0.4330 & -1374.58 & 0.1644 & 2329.54 & 0.0676 & 1224.11 & 0.1490 & 1105.43 & 0.2632 & 2598.68 & 0.0082 \\
\hline 5,10 & 1374.33 & 0.2236 & 237.71 & 0.4381 & 3227.69 & 0.0272 & 2283.55 & 0.0466 & 1136.61 & 0.2854 & 2253.04 & 0.0395 \\
\hline 0,5 & 4013.46 & 0.0112 & 3112.33 & 0.0373 & 4305.87 & 0.0052 & 3404.74 & 0.0302 & 865.87 & 0.3455 & 292.40 & 0.4765 \\
\hline$-5,0$ & 6854.30 & 0.0000 & 5875.57 & 0.0002 & 4616.65 & 0.0024 & 3791.28 & 0.0150 & 825.37 & 0.3137 & -1747.23 & 0.0792 \\
\hline$-10,-5$ & 8723.66 & 0.0000 & 7919.08 & 0.0000 & 4863.00 & 0.0013 & 4219.73 & 0.0107 & 643.27 & 0.2788 & -3586.31 & 0.0069 \\
\hline$-15,-10$ & 9649.68 & 0.0000 & 7727.16 & 0.0000 & 5994.04 & 0.0013 & 4071.51 & 0.0129 & 1922.53 & 0.1363 & -3655.64 & 0.0069 \\
\hline$-20,-15$ & 7437.61 & 0.0003 & 6051.83 & 0.0011 & 6151.21 & 0.0017 & 4279.33 & 0.0119 & 1385.78 & 0.2656 & -1481.91 & 0.2102 \\
\hline$-25,-20$ & 4618.32 & 0.0148 & 4044.56 & 0.0118 & 4679.33 & 0.0118 & 3894.36 & 0.0152 & 573.76 & 0.3826 & 80.28 & 0.4544 \\
\hline$-30,-25$ & 2682.88 & 0.0741 & 2609.11 & 0.0766 & 4594.21 & 0.0060 & 4408.26 & 0.0056 & 185.95 & 0.4363 & 1884.87 & 0.1072 \\
\hline$-35,-30$ & 2792.20 & 0.0717 & 1732.04 & 0.1484 & 5047.46 & 0.0034 & 3864.55 & 0.0083 & 1182.90 & 0.2264 & 2257.67 & 0.0655 \\
\hline$-40,-35$ & 2560.95 & 0.0711 & 1597.18 & 0.1422 & 4972.26 & 0.0040 & 4008.49 & 0.0097 & 963.76 & 0.2713 & 2411.31 & 0.0564 \\
\hline$-45,-40$ & 2449.71 & 0.0804 & 1420.34 & 0.1663 & 5032.67 & 0.0086 & 3779.51 & 0.0258 & 1198.12 & 0.2432 & 2573.52 & 0.0640 \\
\hline$-50,-45$ & 2701.66 & 0.0879 & 2697.81 & 0.0934 & 4126.46 & 0.0300 & 4025.30 & 0.0377 & 94.91 & 0.4008 & 1601.15 & 0.1813 \\
\hline
\end{tabular}

2. The data having a statistically significant difference are mainly distributed near the geographical or geomagnetic equator regions and are more skewed towards the Southern Hemisphere, where the time of the observation data is just summer.

3. Comparing the distribution of data with significant differences in Fig. 4, it is seen that the distribution is $5^{\circ}$ south in geomagnetic latitude, which indicates that this regular distribution of the data with significant differences may be mainly controlled by the geomagnetic latitude, and the regular distribution in terms of the geo- graphical latitude is due to the distribution region in geographical latitude overlapping with regions beside the geomagnetic equator.

4. Table 3 shows that the data differences change from being positive from lower to higher mid-latitudes in the Southern Hemisphere to being negative in the corresponding latitudes in the Northern Hemisphere, just like an arch extending toward the higher latitudinal direction in both hemispheres, as shown in Fig. 5. This regular distribution cannot be a coincidence because although most $p$ values in the mid-latitude regions do not reject 


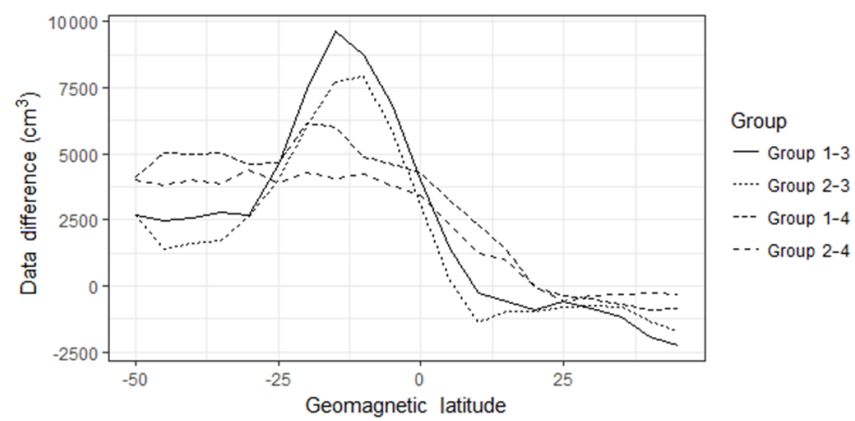

Figure 5. Variations in data differences with geomagnetic latitude.

the null hypothesis of no significant difference between the data observed at different altitudes, the probability that positive differences appear simultaneously in several continuously latitudinal regions (multiplication of the $p$ values in each latitudinal region) is extremely low according to the obtained $p$ values, which indicates an underlying control factor. Regarding all differences in the Northern (winter) Hemisphere being negative, this is the normal attenuation pattern of the F2 layer.

The distribution characteristic, namely that data with significant differences are distributed in the vicinity of the geomagnetic equator, is consistent with the regions where stratification of the F2 layer has been found in many studies, and the stratification phenomenon can exactly explain the electron density at higher altitude being greater than that at lower altitude.

Figure 6 presents all the regular patterns summarized above using the average electron density data of the four groups before and after altitude adjustment in each latitudinal region. The figure shows that the curves of the average electron density data vary with latitude, with the maximum differences being located at about $10^{\circ}$ in the Southern Hemisphere.

Figure 6 shows that the difference between the two groups of data before the adjustment of the orbital altitude, namely Group 1 and 2, is small, while the difference between the two groups after the adjustment, namely Group 3 and 4, is also small. However, when comparing the four groups together, obvious differences between the groups before and after the adjustment are seen in the vicinity of a geographical latitude of $-10^{\circ}$ or a geomagnetic latitude of $-15^{\circ}$. Moreover, the difference is more pronounced in the Southern Hemisphere than in the Northern Hemisphere. Although the greater data fluctuations in the summer Southern Hemisphere are a cause of this phenomenon, the regular distribution cannot be explained by random fluctuation in the data.

\subsection{Reference comparisons}

To further demonstrate that the phenomenon found above is caused by non-random factors, several sets of data other than the abovementioned data are constructed to compare whether the same regular distribution patterns can be found.

\subsubsection{Descending data for the same period}

The permutation test results of descending data, data recorded during the day, are calculated according to the grouping information in Table 1. The results show that there are both cases of significant differences and insignificant differences between the data observed at different altitudes and between the data observed at same altitudes. Variations in the average electron density with latitude are given in Fig. 7 . The figure clearly shows that the observation data for the same altitude during the day fluctuate greatly, and there are no consistent regularities among different data groups. Therefore, although there are cases in which a higher altitude has higher electron density, a definite conclusion cannot be drawn from these descending data.

\subsubsection{Ascending data in different periods}

Besides the above analysis, groups of reference data are also calculated to further confirm that the regular distributions in Fig. 4 are not accidental. Because there were small geomagnetic storms in January 2007 and 2008, only data in 2009 and 2010 are used here for comparison. Data groups, with the same geomagnetic and grouping conditions and using the ascending data (data observed during nighttime) for 2009 and 2010, are calculated using the permutation test method. Figures 8 and 9 show the variations in ascending electron density data with geographical or geomagnetic latitude using the data recorded in 2009 and 2010 respectively; no obvious differences are found from these data. Therefore, the significant differences shown in Fig. 4 are not coincidental.

\section{Discussion}

We conclude from the above data analysis that the phenomenon of the in situ electron density observed at higher altitude being greater than that observed at lower altitude and the significant differences being distributed regularly in the vicinity of the geomagnetic equator on a global scale is the stratification phenomenon of the F2 layer. Although the data were not recorded at the same time, the data variation can be neglected because the time interval is short and observing conditions are similar.

According to the data grouping and calculation method, if the phenomenon is only due to random data fluctuation, the possibility that this phenomenon appears only for data recorded at different altitudes and at several latitudinal regions in the vicinity of the geomagnetic equator at the same 

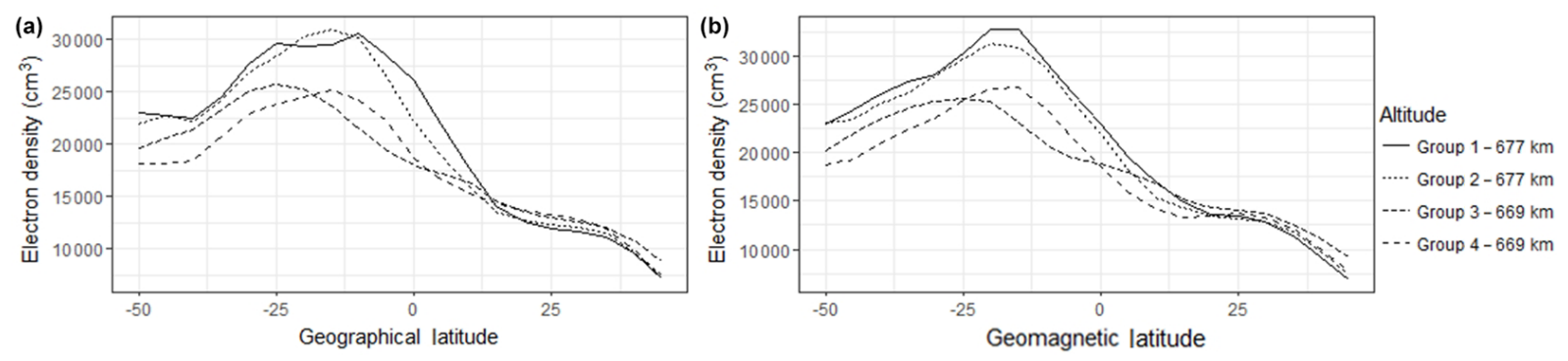

Figure 6. Variation in the average ascending electron density with latitude.
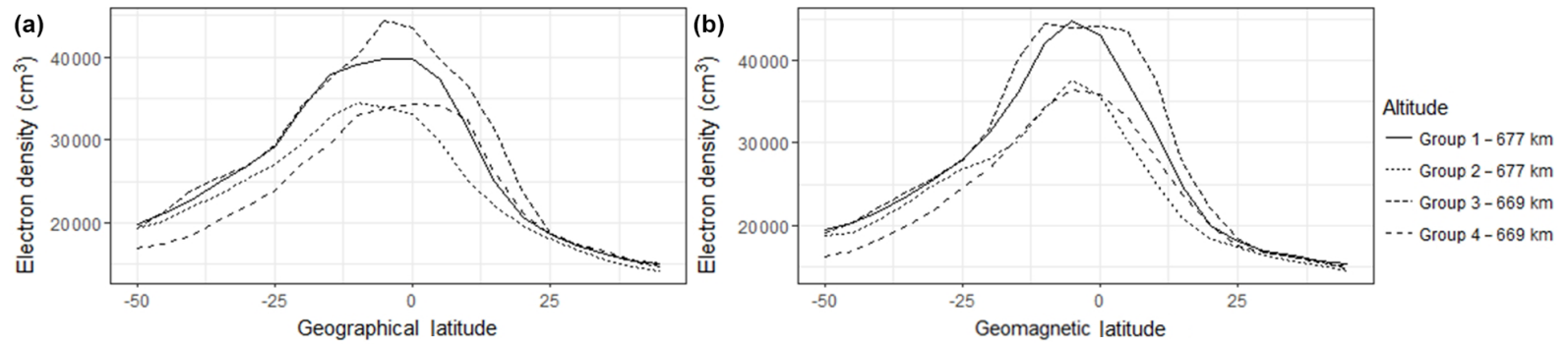

Figure 7. Variation in the average descending electron density with latitude.

time is extremely low. Moreover, the same regular distribution from data recorded at other times with similar grouping conditions cannot be observed. The possibility that the regular data distribution is due to random factors can therefore be excluded definitely.

In addition, the significant difference between two data groups before and after the altitude adjustment near the geomagnetic equator region indicates that most data in the 36 cells in each latitudinal region have a significant difference. It is thus deduced from the data that the stratification phenomenon in the F2 layer covers a large longitudinal area near the geomagnetic equator region. This is different from the conclusion of the studies (Balan et al., 1998; Rama Rao et al., 2005; Zhao et al., 2011a) in which the phenomenon can only be observed at special longitudes, which may be due to the fact that the peak of the stratification is less than that of the F2 layer in most of the longitudinal area for most of the time and thus invisible to the ground-based observation.

In fact, the stratification phenomenon has been observed at many locations using the ionosonde, e.g., Brazil (Balan et al., 1997, 1998, 2000; Batista et al., 2002; Jenkins et al., 1997), southeastern Asia (Hsiao et al., 2001; Lynn et al., 2000), India (Rama Rao et al., 2005; Thampi et al., 2005), and China (Jiang et al., 2015), illustrating that the stratification phenomenon is distributed across a large longitudinal area in spite of the scatter discoveries. The study of Zhao et al. (2011b) using long-term satellite-based radio occultation observations also showed that the stratification is distributed in all longitudinal areas along the magnetic equator. The results obtained from the in situ data are thus in accordance with the results of those studies and further prove that this phenomenon may be continuously distributed along the longitudinal direction. The global-scale in situ electron density data of the DEMETER satellite observed in a short time provide an opportunity to study the distribution features of the stratification phenomenon, which are difficult to detect through scattered ground-based or satellite-based sounding data.

Section 3 showed that the recording time of the data used in this study, namely the time of the stratification, happened to coincide with the downward cycle of the 23rd solar cycle, when the solar activity was relatively low. The season of stratification found in the data in this study coincided with summer in the Southern Hemisphere, and the stratification was almost entirely located in the Southern Hemisphere in terms of the geomagnetic latitude. These spatial and temporal distribution characteristics, distinct on the summer side of low solar activity, are exactly the same as those of the F2-layer stratification phenomenon obtained in many studies (Balan et al., 1998; Batista et al., 2002; Nayak et al., 2014; Rama Rao et al., 2005; Sharma and Raghavarao, 1989).

As for the local time at which stratification occurs, many studies have suggested that the stratification phenomenon mainly occurs during the day, just as Balan et al. (1998) reported that the F3 layer occurs mainly during the morningnoon period, owing to the combined effect of the upward $\boldsymbol{E} \times \boldsymbol{B}$ drift and neutral wind that provides upward plasma drifts at and above the F2 layer. However, more and more studies have confirmed the existence of nighttime stratification. Zhao et al. (2011a) studied the post-sunset stratification phenomenon and suggested that the sunset F3 layer should be distinguished from the traditional morning-noon F3 layer. 

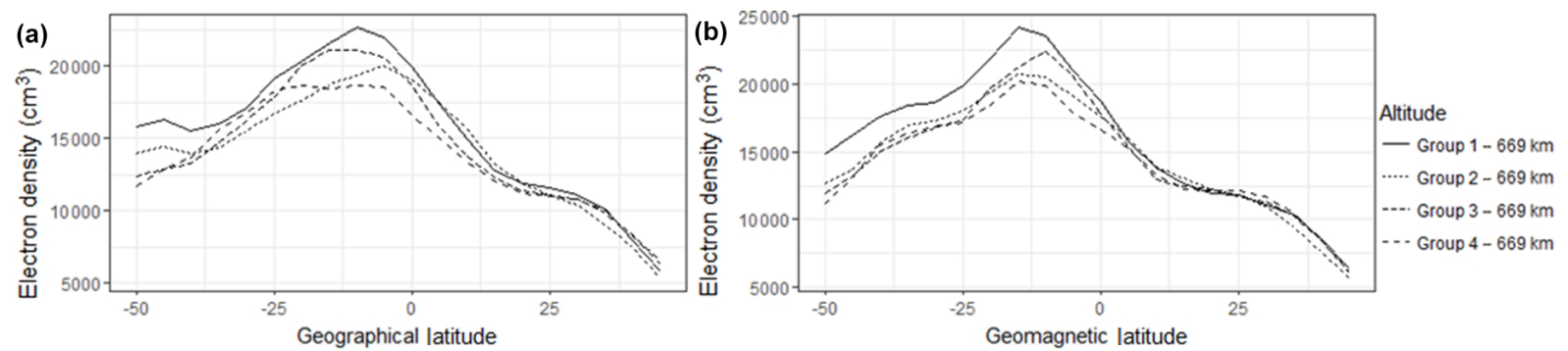

Figure 8. Variation in the ascending electron density with latitude, obtained using data for 2009.
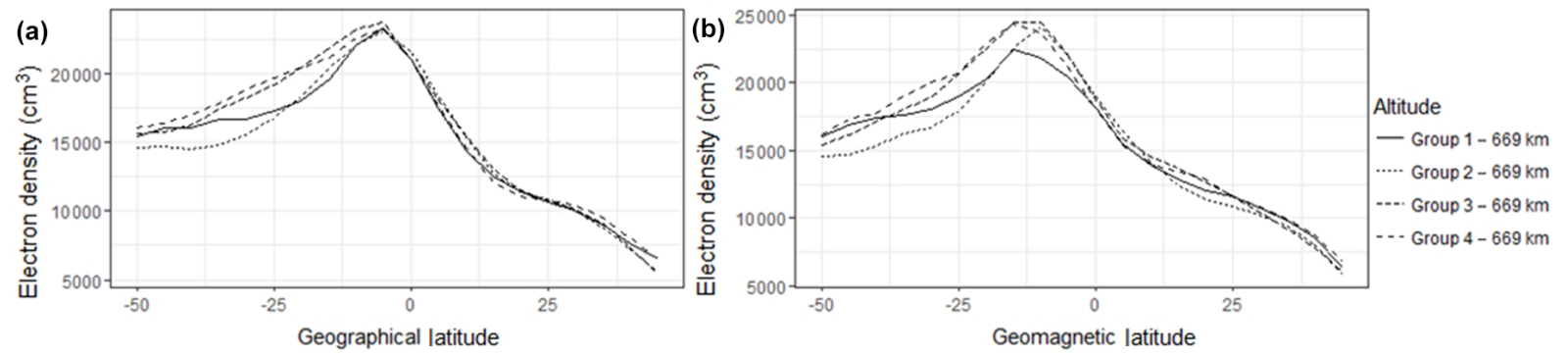

Figure 9. Variation in the ascending electron density with latitude, obtained using data for 2010.

Lockwood and Nelms (1964) suggested that the stratification of the F layer can be observed until about midnight local time using the topside sounder data of the ionogram aboard the Alouette satellite. Karpachev et al. (2012) examined the large dataset of IK-19 and found that the F3 layer can permanently exist until 02:00-03:00 LT. Nevertheless, the F3 layer is rarely recorded at night.

Depuev and Pulinets (2001) also found midnight stratification and showed that the critical frequency of the nocturnal F3 layer is always essentially lower than $f_{0} \mathrm{~F} 2$. It is thus impossible to observe midnight stratification from the bottom side. They also reported that the real peak height $(h m \mathrm{~F} 3)$ of the F3 layer defined by electron density profiles varied from 670 to $730 \mathrm{~km}$. Rama Rao et al. (2005) pointed out that the altitude of the F3 layer is high at the magnetic equator (600$700 \mathrm{~km})$. The altitude of the stratification in these studies is almost the same as the altitudes of the in situ data used in this paper.

Klimenko et al. (2012) suggested that the formation mechanism of additional layers in the equatorial ionosphere is due to the action of the non-uniformity in the height of the zonal electric field at the geomagnetic equator and can happen at any time, which can explain the occurrences of the F3 layer and multiple layers at different local times, especially at night.

An interesting point which has not been discussed in earlier studies is that all differences in each latitudinal region on the summer hemisphere are positive, though some do not pass a significance test. This consistent distribution cannot be obtained if data fluctuate randomly. We therefore speculate that this feature may be related to the stratification phe- nomenon, and small stratification may exist in the summer hemisphere a short distance away from the traditional geomagnetic equator region of stratification.

Summarizing the above discussions, we believe that the results obtained in this paper are the stratification phenomenon in the ionospheric F2 layer, and the proposed method is effective. The results of this method indicate that the stratification phenomenon may extend to a larger area in the summer hemisphere, but it is difficult to detect because the differences are small. The distribution features obtained by the data analytic results also indicate that the stratification phenomenon is more complex than what has been found previously.

\section{Conclusion}

To compare the in situ electron density data observed by the DEMETER Satellite at different altitudes, a statistical method, using the permutation resampling skill, is adopted and used to carry out the data comparison and analysis work. The results of 10000 permutation tests, using the ascending data (data observed during nighttime) obtained before and after the altitude adjustment, show that there are significant differences between data recorded at different altitudes near the geomagnetic equator, but no significant differences can be found from the multiple reference datasets. The stratification phenomenon can explain the regular distribution patterns summarized from the data analytic results. In addition, the location, altitude, season, and local time of this phenomenon are accordance with the results of many studies on the F2-layer stratification phenomenon. We therefore be- 
lieve that the significant difference between the observations of the DEMETER satellite at different altitudes is the stratification phenomenon, and the proposed method is effective and applicable to similar data analytic studies.

Some features of the stratification phenomenon can also be summarized from the data analysis results.

The possible stratification phenomenon is found from the nighttime data but cannot be obtained from the corresponding daytime data, though many studies have pointed out that this phenomenon occurs mainly during the day, which implies the nighttime stratification may be a permanent phenomenon.

The phenomenon can occur in most longitudinal regions, which is not in accordance with the finding of studies that the phenomenon can only appear in special longitudinal regions. This may be due to the peak of the stratification being less than $f_{0} \mathrm{~F} 2$ in most longitudinal regions for most of the time.

The significance of differences decreases with latitude away from the geomagnetic equator, indicating that the stratification is just as an arch along the latitude.

Data differences, all of which are positive at lower to higher mid-latitudes in the summer hemisphere, indicate that the latitudinal extent of the stratification phenomenon is much larger in the summer hemisphere than the winter hemisphere and that small stratification may exist away from the traditional stratification region. The stratification phenomenon is more complex than what has previously been found.

Data availability. The electron density data used in this paper are available from the DEMETER Data Server (http://demeter. cnrs-orleans.fr/, last access: 25 July 2019).

Author contributions. Xiuying Wang arranged this study, including experiment design and data analysis. Dehe Yang and Dapeng Liu collected the data used in this paper. Wei Chu did some calculation work.

Competing interests. The authors declare that they have no conflict of interest.

Acknowledgements. This work is supported by the National Key R\&D Program of China under grant no. 2018YFC1503505, by the Beijing Natural Science Foundation under grant no. 8184091, and by the Foundation of the Institute of Crustal Dynamics, CEA, under grant no. ZDJ2017-20. The electron density data used in this paper are available from the DEMETER Data Server (http: //demeter.cnrs-orleans.fr/, last access: 25 July 2019).

Financial support. This research has been supported by the National Key R\&D Program of China (grant no. 2018YFC1503505), by the Beijing Natural Science Foundation (grant no. 8184091), and by the Foundation of the Institute of Crustal Dynamics, CEA (grant no. ZDJ2017-20).

Review statement. This paper was edited by Dalia Buresova and reviewed by Angelo De Santis and Galina Gordienko.

\section{References}

Balan, N. and Bailey, G. J.: Equatorial plasma fountain and its effects: Possibility of an additional layer, J. Geophys. Res., 100, 21421-21432, https://doi.org/10.1029/95JA01555, 1995.

Balan, N., Bailey, G. J., Abdu, M. A., Oyama, K. I., Richards, P. G., Macdougall, J., and Batista, I. S.: Equatorial plasma fountain and its effects over three locations: Evidence for an additional layer, the F3 layer, J. Geophys. Res., 102, 2047-2056, https://doi.org/10.1029/95JA02639, 1997.

Balan, N., Batista, I. S., Abdu, M. A., MacDougall, J., and Bailey, G. J.: Physical mechanism and statistics of occurrence of an additional layer in the equatorial ionosphere, J. Geophys. Res.Atmos., 103, 29169-29182, https://doi.org/10.1029/98JA02823, 1998.

Balan, N., Batista, I. S., Abdu, M. A., Bailey, G. J., Watanabe, S., MacDougall, J., and Sobral, J. H. A.: Variability of an additional layer in the equatorial ionosphere over Fortaleza, J. Geophys. Res.-Space, 105, 10603-10613, https://doi.org/10.1029/1999ja000020, 2000.

Batista, I. S., Abdu, M. A., McDougall, J., and Souza, J. R.: Long term trends in the frequency of occurrence of the F3 layer over Fortaleza, J. Atmos. Sol.-Terr. Phy., 64, 1409-1412, https://doi.org/10.1016/S1364-6826(02)00104-9, 2002.

Depuev, V. H. and Pulinets, S. A.: Intercosmos-19 observations of an additional topside ionization layer: The F3-layer, Adv. Space. Res., 27, 1289-1292, https://doi.org/10.1016/S02731177(01)00205-8, 2001.

Durstenfeld, R.: Algorithm 235:Random permutation, Commun. ACM, 7, 420, https://doi.org/10.1145/364520.364540, 1964.

Heisler, L. H.: The anomalous ionospheric stratification F1.5, J. Atmos. Terr. Phys., 24, 483-489, https://doi.org/10.1016/00219169(62)90212-X, 1962.

Hesterberg, T., Monaghan, S., Moore, D. S., Clipson, A., and Epstein, R.: Bootstrap Methods and Permutation Tests: Companion Chapter 18 to the Practice of Business Statistics, W.H. Freeman and Company, New York, 2003.

Hsiao, C. C., Liu, J. Y., Tsunoda, R. T., Fukao, S., Saroso, S., Nozaki, K., Badillo, V. L., Berkey, F. T., Chen, S. W., and Yamamoto, M.: Evidence for the geographic control of additional layer formation in the low-latitude ionosphere, Adv. Space. Res., 27, 1293-1297, https://doi.org/10.1016/S0273-1177(01)00206$\mathrm{X}, 2011$.

Jenkins, B., Bailey, G. J., Abdu, M. A., Batista, I. S., and Balan, N.: Observations and model calculations of an additional layer in the topside ionosphere above Fortaleza, Brazil, Ann. Geophys., 15, 753-759, https://doi.org/10.1007/s00585-997-0753-3, 1997.

Jiang, C. H., Yang, G. B., Deng, C, Zhou, C., Zhu, P., Yokoyama, T., Song, H., Lan, T., Ni, B. B., Zhao, Z. Y., and Zhang, Y. N.: Simultaneous observation of F2 layer stratification 
and spread $\mathrm{F}$ at postmidnight over a northern equatorial anomaly region, J. Geophys. Res.-Space, 120, 10979-10991, https://doi.org/10.1002/2015JA021861, 2015.

Karpachev, A. T., Klimenko, M. V., Klimenko, V. V., Zhbankov, G. A., and Telegin, V. A.: Latitudinal structure of the equatorial F3 layer based on Intercosmos-19 topside sounding data, J. Atmos. Sol.-Terr. Phy., 77, 186-193, https://doi.org/10.1016/j.jastp.2011.12.018, 2012.

Klimenko, M. V. and Klimenko, V. V.: Mechanisms of stratification of the F2 layer and formation of the F3 and G layers in the equatorial ionosphere, Geomagn. Aeronomy, 52, 321-334, https://doi.org/10.1134/S0016793212030097, 2012.

Lagoutte, D., Brochot, J. Y., Carvalho, D., Elie, D., Harivelo, F., Hobara, Y., Madrias, L., Parrot, M., Pincon, J. L., Berthelier, J. J., Peschard, D., Seran, E., Gangloff, M., Sauvaud, J. A., Lebreton, J. P., Stverak, S., Travnicek, P., Grygorczuk, J., Slominski, J., Wronowski, R., Barbier, S., Bernard, P., Gaboriaud, A., and Wallut, J. M.: The DEMETER Science Mission Centre, Planet. Space Sci., 54, 428-440, https://doi.org/10.1016/j.pss.2005.10.014, 2006.

Lebreton, J. P., Stverak, S., Travnicek, P., Maksimovic, M., Klinge, D., Merikallio, S., Lagoutte, D., Poirier, B., Blelly, P. L., Kozacek, Z., and Salaquarda, M.: The ISL Langmuir probe experiment processing onboard DEMETER: Scientific objectives, description and first results, Planet. Space Sci., 54, 472-486, https://doi.org/10.1016/j.pss.2005.10.017, 2006.

Lockwood, G. E. K. and Nelms, G. L.: Topside sounder observations of the equatorial anomaly in the $75 \mathrm{~W}$ longitude zone, J. Atmos. Terr. Phys., 26, 569-580, https://doi.org/10.1016/00219169(64)90188-6, 1964.

Lynn, K. J. W., Harris, T. J., and Sjarifudin, M.: Stratification of the F2 layer observed in Southeast Asia, J. Geophys. Res., 105, 27147-27156, https://doi.org/10.1029/2000JA900056, 2000.

Nayak, C. K., Yadav, V., Kakad, B., Sripathi, S., Emperumal, K., Pant, T. K., Bhattacharyya, A., and Jin, S. G.: Peculiar features of ionospheric F3 layer during prolonged solar minimum (2007-2009), J. Geophys. Res.-Space, 119, 8685-8696, https://doi.org/10.1002/2014JA020135, 2014.

Raghavarao, R. and Sivaraman, M.R.: Ionization ledges in the equatorial ionosphere, Nature, 249, 331-332, https://doi.org/10.1038/249331a0, 1974.

Rama Rao, P. V. S., Niranjan, K., Prasad, D. S. V. V. D., Brahmanandam, P. S., and Gopikrishna S.: Features of additional stratification in ionospheric F2 layer observed for half a solar cycle over Indian low latitudes, J. Geophys. Res., 110, A04307, https://doi.org/10.1029/2004JA010646, 2005.

Sayers, J., Rothwell, P., and Wager J. H.: Field Aligned Strata in the Ionization above the Ionspheric F2 Layer, Nature, 198, 230-233, https://doi.org/10.1038/198230a0, 1963.
Sen, H. Y.: Stratification of the F2 layer of the ionosphere over Singapore, J. Geophys. Res., 54, 363-366, https://doi.org/10.1029/jz054i004p00363, 1949.

Sharma, P. and Raghavarao, R.: Simultaneous occurrence of ionization ledge and counterelectrojet in the equatorial ionosphere: observational evidence and its implications, Can. J. Phys., 67, 166-172, https://doi.org/10.1139/p89-028, 1989.

Skinner, N. J., Brown, R. A., and Wright, R. W.: Multiple stratification of the F-layer at Ibadan, J. Atmos. Terr. Phys., 5, 92-100, https://doi.org/10.1016/0021-9169(54)90013-6, 1954.

Tardelli, A., Fagundes, P. R., Pezzopane, M., Venkatesh, K., and Pillat, V. G.: Seasonal and solar activity variations of F3 layer and quadruple stratification (StF-4) near the equatorial region, J. Geophys. Res.-Space, 121, 12116-12125, https://doi.org/10.1002/2016JA023580, 2016.

Thampi, S. V., Ravindran, S., Devasia, C. V., Pant, T. K., Sreelatha, P., and Sridharan, R.: First observation of topside ionization ledges using radio beacon measurements from low Earth orbiting satellites, Geophys. Res. Lett., 32, L11104, https://doi.org/10.1029/2005GL022883, 2005.

Uemoto, J., Ono, T., Kumamoto, A., and Iizima, M.: Ionization ledge structures observed in the equatorial anomaly region by using PPS system on-board the Ohzora (EXOS-C) satellite, Earth Planets Space, 56, e21-e24, https://doi.org/10.1186/BF03352524, 2004.

Uemoto, J., Ono, T., Kumamoto, A., and Iizima, M.: Statistical analysis of the ionization ledge in the equatorial ionosphere observed from topside sounder satellites, J. Atmos. Terr. Phys., 68, 1340 1351, https://doi.org/10.1016/j.jastp.2006.05.015, 2006.

Uemoto, J., Ono, T., Maruyama, T., Saito, S., Iizima, M., and Kumamoto, A.: Magnetic conjugate observation of the F3 layer using the SEALION ionosonde network, Geophys. Res. Lett., 34, L02110, https://doi.org/10.1029/2006g1028783, 2007.

Zain, A. F. M., Abdullah, S., Homam, M. J., Seman, F. C., Abdullah, M., and Ho, Y. H.: Observations of the F3-layer at equatorial region during 2005, J. Atmos. Sol.-Terr. Phy., 70, 918-925, https://doi.org/10.1016/j.jastp.2007.12.002, 2008.

Zhao, B., Wan, W., Reinisch, B., Yue, X., Le, H., Liu, J., and Xiong, B.: Features of the F3 layer in the lowlatitude ionosphere at sunset, J. Geophys. Res., 116, A01313, https://doi.org/10.1029/2010JA016111, 2011a.

Zhao, B., Wan, W., Yue, X., Liu, L., Ren, Z., He, M., and Liu, J.: Global characteristics of occurrence of an additional layer in the ionosphere observed by COSMIC/FORMOSAT-3, Geophys. Res. Lett., 38, L02101, https://doi.org/10.1029/2010GL045744, $2011 b$. 